\title{
Estimation and Control of Battery Electrochemistry Models: A Tutorial
}

\author{
Scott J. Moura
}

\begin{abstract}
This paper presents a tutorial on estimation and control problems for battery electrochemistry models. We present a background on battery electrochemistry, along with a comprehensive electrochemical (EChem) model. EChem models present a remarkably rich set of control-theoretic questions involving model reduction, state \& parameter estimation, and optimal control. We discuss fundamental systems and controls challenges, and then present opportunities for future research.
\end{abstract}

\section{INTRODUCTION}

Batteries are ubiquitous - they exist in our smart phones, laptops, electric vehicles (EVs), and electric grids. Energy storage is a critical enabling technology for designing sustainable energy systems. Although battery materials science has seen rapid advances [1], batteries are chronically underutilized and conservatively designed [2]. Consumers purchase batteries with 20-50\% excess energy capacity, leading to added weight, volume, and upfront cost. Intelligent battery control can lead to faster charge times, increased energy and power capacity, as well as a longer life. The key to realizing such advanced battery management systems is electrochemical model based controls. These electrochemical models, however, contain a multitude of fundamental controltheoretic challenges, yielding a rich opportunity for systems and controls researchers.

\section{A. Background \& Fundamentals of Battery Electrochemistry}

Italian physicist Alessandro Volta invented the first battery cell in 1800. The so-called voltaic pile consisted of two metals in series, zinc and copper, coupled by a sulphuric acid electrolyte. Volta constructing this system in response to experiments performed by his colleague Luigi Galvani, who was fascinated by the interaction between electricity and biological nervous systems. During an experiment, Galvani discovered that a dead frog's legs would kick when connected to two dissimilar metals. Galvani conjectured the energy originated within the animal, coining the phenomenon "animal electricity." Volta believed the different metals caused this behavior, and proved his hypothesis true with the voltaic pile [3]. Thus, the electrochemical battery cell was born.

A battery converts energy between the chemical and electrical domains through oxidation-reduction reactions. It consists of two dissimilar metals (electrodes) immersed in an

This work was supported in part by the National Science Foundation under Grant No. 1408107 and the Advanced Research Projects AgencyEnergy, U.S. Department of Energy, under Award Number DE-AR0000278.

S. J. Moura is with the Energy, Controls, and Applications Lab (eCAL) in Civil and Environmental Engineering at the University of California, Berkeley in Berkeley, California, 94720, USA smoura@berkeley . edu

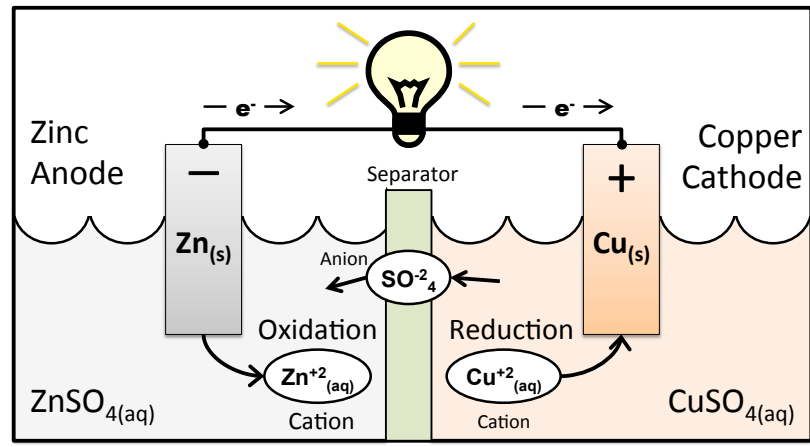

Fig. 1. An example zinc-copper Galvanic (or Voltaic) cell demonstrating the principles of operation for an electrochemical cell.

electrolyte, exemplified by the zinc-copper Galvanic cell in Fig. 1. The cathode and anode materials are jointly selected to have a large electrochemical potential between each other. This creates the desired electrochemical energy storage property. The electrodes are electrically isolated by a separator. Hence, conservation of charge forces electrons through an external circuit, powering a connected device, while cations flow between the electrodes within the electrolyte.

Electrode and electrolyte materials are selected for their voltage, charge capacity, weight, cost, manufacturability, etc. For example, lithium-ion cells are attractive in mobile applications because lithium is the lightest $(6.94 \mathrm{~g} / \mathrm{mol})$ and most electropositive $(-3.01 \mathrm{~V}$ vs. standard hydrogen electrode) metal in the periodic table. Lead acid cells feature heavier electrodes $(\mathrm{Pb}$ and $\mathrm{PbO} 2)$, yet provide high surge currents at cost effective prices. Lithium-air batteries feature cathodes that couple electrochemically with atmospheric oxygen, thus producing energy densities that rival gasoline fuel. In battery energy management, we are interested in maximizing performance and longevity. This requires a detailed understanding of the underlying electrochemistry. However, the electrochemical state variables are not directly measurable. At best, one can measure voltage, current, and temperature only. Consequently, modeling and control are necessary to extract the full potential from batteries.

\section{B. Estimation and Control Problem Statements}

Figure 2 outlines an electrochemical model based control system, comprised of a state estimator, parameter estimator, and controller. Next, we provide precise problem statements for each control-theoretic task.

1) State-of-Charge (SOC) Estimation: $\mathrm{SOC}$ indicates the quantity of lithium within each electrode's solid phase. It is analogous to a fuel tank level, since it represents the 


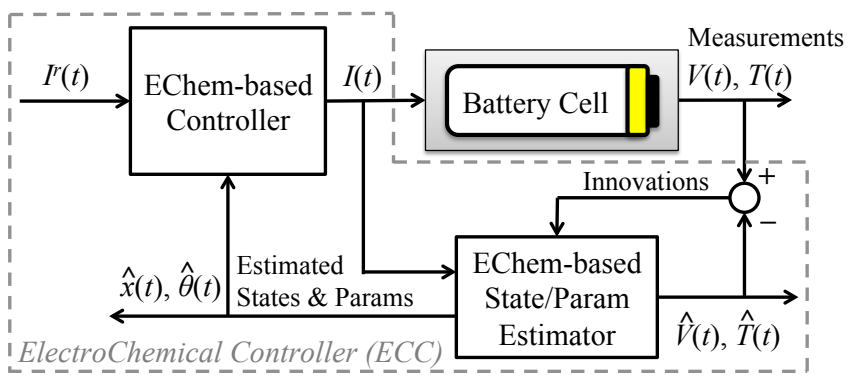

Fig. 2. Block diagram for ElectroChemical Control (ECC) System, comprised of a battery cell, state/parameter estimator, and controller.

stored electrochemical energy. Unlike fuel tanks, SOC is not measurable and must be estimated. This can be directly cast as a state estimation problem.

The State Estimation Problem: Given measurements of current $I(t)$, voltage $V(t)$, and temperature $T(t)$, estimate the concentration of lithium in each electrode's solid phase material. Optionally, one may estimate other electrochemical variables of interest, such as electrolyte concentration and overpotentials (see Section II-B).

The key challenges for state estimation are lack of complete observability and model nonlinearities.

2) State-of-Health (SOH) Estimation: Battery $\mathrm{SOH}$ metrics indicate a battery's relative age. The two most common $\mathrm{SOH}$ metrics are charge capacity fade and impedance rise (i.e. power fade). Charge capacity fade indicates how charge capacity has decreased relative to its nameplate value, e.g. a 2 Ah cell may hold 1.6 Ah after two years of use. Power capacity fade indicates how power capacity has decreased relative to its nameplate value, e.g. a fresh cell may provide $360 \mathrm{~W}$ of power for 10 seconds, but only $300 \mathrm{~W}$ after two years of use. Gradual changes in SOH metrics are directly related to changes in the mathematical model parameters.

The Parameter Identification Problem: Given measurements of current $I(t)$, voltage $V(t)$, and temperature $T(t)$, estimate uncertain parameters related to $\mathrm{SOH}$, such as cyclable lithium, solid-electrolyte interface resistance, and volume fractions.

The key challenges for electrochemical models include parametric modeling, nonlinear parameter identifiability, and persistent excitation.

3) Controlled Charging/Discharging: In many applications, additional capacity is added to mitigate cell imbalance, capacity/power fade, thermal effects, and estimation errors. This leads to larger, heavier, and more costly batteries than necessary. Electrochemical control alleviates oversizing by safely operating batteries near their physical limits. Today, operational limits are defined by what can be measured - voltage, current, and temperature. Battery degradation, however, is more closely related to limits on immeasurable electrochemical states, such as overpotentials and surface concentrations [2]. We seek a paradigm-shifting architecture that expands the operating envelope by constraining internal electrochemical states instead of measured values such as voltage, current, and temperature (see Fig. 3).

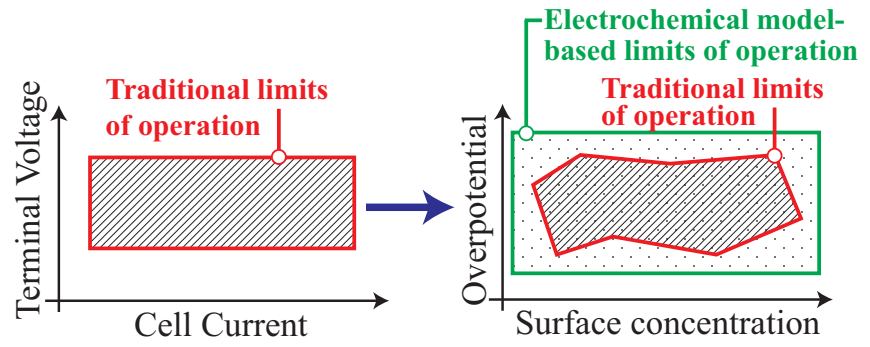

Fig. 3. An electrochemical model-based control architecture constrains electrochemical state variables, as opposed to measurable outputs. This expands the operational envelope and ensures safety.

The Constrained Control Problem: Given measurements of current $I(t)$, voltage $V(t)$, and temperature $T(t)$, control current such that critical electrochemical variables are maintained within safe operating constraints.

\section{MATHEMATICAL MODELING}

\section{A. Background}

Electrochemical (EChem) models capture the spatiotemporal dynamics of lithium-ion concentration, electric potential, and intercalation kinetics. Most models in the battery controls literature are derived from the Doyle-FullerNewman (DFN) model [4], which is based upon porous electrode and concentrated solutions theory. Figure 4 shows a cross section of the layers described in Fig. 1.

At full charge the majority of lithium exists within the anode solid phase particles, typically a lithiated carbon $\mathrm{Li}_{x} \mathrm{C}_{6}$. These particles are idealized as spherically symmetric. During discharge, lithium diffuses from the interior to the surface of these porous spherical particles. At the surface an electrochemical reaction separates lithium into a positive lithium ion and electron (1). Next, the lithium ion migrates from the anode, through the separator, and into the cathode. Since the separator is an electrical insulator, the corresponding electron travels through an external circuit, powering the connected device. The lithium ion and electron meet at the cathode particle surface, typically a lithium metal oxide $\mathrm{LiMO}_{2}$, and undergo the reverse electrochemical reaction according to (2).

$$
\begin{aligned}
\mathrm{Li}_{x} \mathrm{C}_{6} & \rightleftharpoons \mathrm{C}_{6}+x \mathrm{Li}^{+}+x \mathrm{e}^{-}, \\
\mathrm{Li}_{(1-x)} \mathrm{MO}_{2}+x \mathrm{Li}^{+}+x \mathrm{e}^{-} & \rightleftharpoons \mathrm{LiMO}_{2} .
\end{aligned}
$$

The resultant lithium atom diffuses into the interior of the cathode's spherical particle. This entire process is reversible by applying sufficient potential across the current collectors - rendering an electrochemical storage device. In addition to lithium migration, this model captures the spatial-temporal dynamics of internal potentials, electrolyte current, and current density between the solid and electrolyte phases.

\section{B. Doyle-Fuller-Newman Model}

We consider the Doyle-Fuller-Newman (DFN) model in Fig. 4 to predict the evolution of lithium concentration in the solid $c_{s}^{ \pm}(x, r, t)$, lithium concentration in the electrolyte 


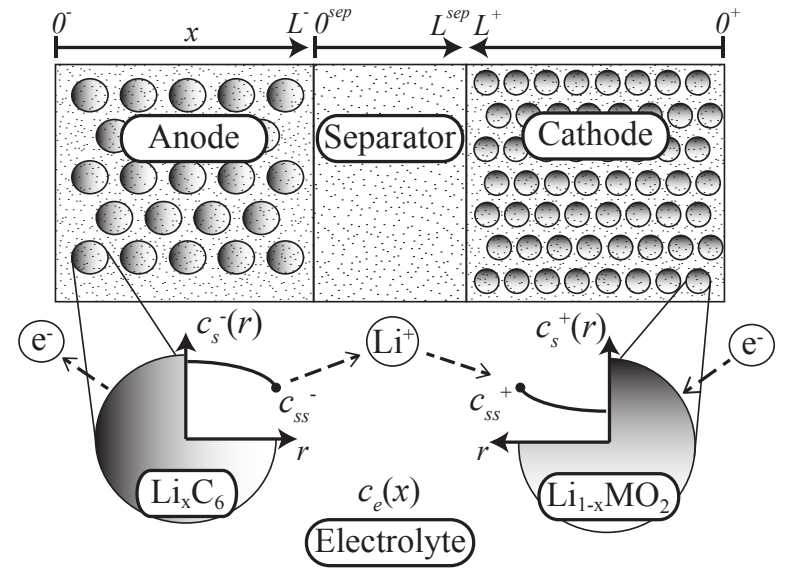

Fig. 4. Schematic of the Doyle-Fuller-Newman model [4]. The model considers two phases: the solid and electrolyte. In the solid, states evolve in the $x$ and $r$ dimensions. In the electrolyte, states evolve in the $x$ dimension only. The cell is divided into three regions: anode, separator, and cathode.

$c_{e}(x, t)$, solid electric potential $\phi_{s}^{ \pm}(x, t)$, electrolyte electric potential $\phi_{e}(x, t)$, ionic current $i_{e}^{ \pm}(x, t)$, molar ion fluxes $j_{n}^{ \pm}(x, t)$, and bulk cell temperature $T(t)[4]$. The governing equations are given by

$$
\begin{aligned}
& \frac{\partial c_{s}^{ \pm}}{\partial t}(x, r, t)=\frac{1}{r^{2}} \frac{\partial}{\partial r}\left[D_{s}^{ \pm} r^{2} \frac{\partial c_{s}^{ \pm}}{\partial r}(x, r, t)\right], \\
& \frac{\partial c_{e}}{\partial t}(x, t)=\frac{\partial}{\partial x}\left[D_{e}\left(c_{e}\right) \frac{\partial c_{e}}{\partial x}(x, t)+\frac{1-t_{c}^{0}}{\varepsilon_{e} F} i_{e}^{ \pm}(x, t)\right], \\
& \frac{\partial \phi_{s}^{ \pm}}{\partial x}(x, t)=\frac{i_{e}^{ \pm}(x, t)-I(t)}{\sigma^{ \pm}}, \\
& \frac{\partial \phi_{e}}{\partial x}(x, t)=-\frac{i_{e}^{ \pm}(x, t)}{\kappa\left(c_{e}\right)}+\frac{2 R T}{F}\left(1-t_{c}^{0}\right) \\
& \times\left(1+\frac{d \ln f_{c / a}}{d \ln c_{e}}(x, t)\right) \frac{\partial \ln c_{e}}{\partial x}(x, t), \\
& \frac{\partial i_{e}^{ \pm}}{\partial x}(x, t)=a_{s} F j_{n}^{ \pm}(x, t), \\
& j_{n}^{ \pm}(x, t)=\frac{1}{F} i_{0}^{ \pm}(x, t)\left[e^{\frac{\alpha_{a} F}{R T} \eta^{ \pm}(x, t)}-e^{-\frac{\alpha_{c} F}{R T} \eta^{ \pm}(x, t)}\right]
\end{aligned}
$$

$$
\begin{aligned}
\rho^{\mathrm{avg}} c_{P} \frac{d T}{d t}(t) & =h_{\text {cell }}\left[T_{\text {amb }}(t)-T(t)\right]+I(t) V(t) \\
& -\int_{0^{-}}^{0^{+}} a_{s} F j_{n}(x, t) \Delta T(x, t) d x,
\end{aligned}
$$

where $D_{e}, \kappa, f_{c / a}$ are functions of $c_{e}(x, t)$ and

$$
\begin{aligned}
i_{0}^{ \pm}(x, t)= & k^{ \pm} \sqrt{c_{s s}^{ \pm}(x, t) c_{e}(x, t)\left(c_{s, \max }^{ \pm}-c_{s s}^{ \pm}(x, t)\right)} \\
\eta^{ \pm}(x, t)= & \phi_{s}^{ \pm}(x, t)-\phi_{e}(x, t) \\
& -U^{ \pm}\left(c_{s s}^{ \pm}(x, t)\right)-F R_{f}^{ \pm} j_{n}^{ \pm}(x, t), \\
c_{s s}^{ \pm}(x, t)= & c_{s}^{ \pm}\left(x, R_{s}^{ \pm}, t\right) \\
\Delta T(x, t)= & U^{ \pm}\left(\bar{c}_{s}^{ \pm}(x, t)\right)-T(t) \frac{\partial U^{ \pm}}{\partial T}\left(\bar{c}_{s}^{ \pm}(x, t)\right) \\
\bar{c}_{s}^{ \pm}(x, t)= & \frac{3}{\left(R_{s}^{ \pm}\right)^{3}} \int_{0}^{R_{s}^{ \pm}} r^{2} c_{s}^{ \pm}(x, r, t) d r
\end{aligned}
$$

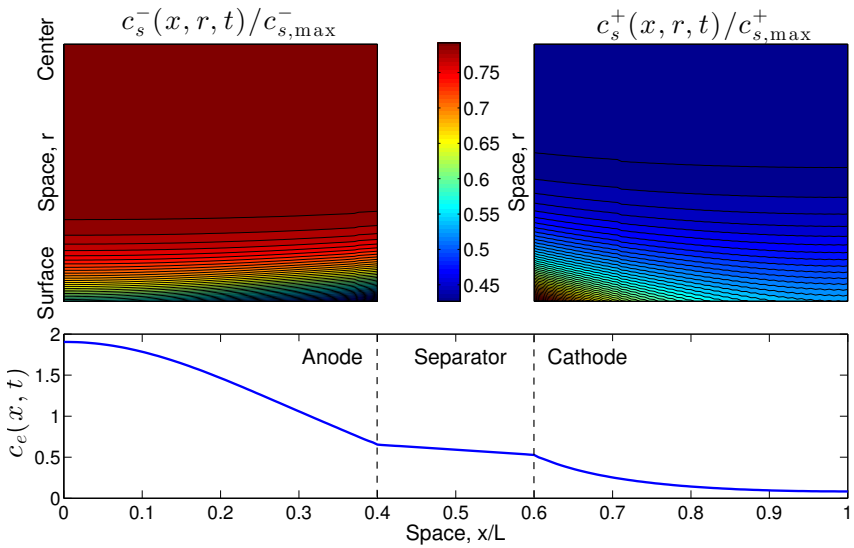

Fig. 5. Solid $c_{s}^{ \pm}(x, r, t)$ and electrolyte $c_{e}(x, t)$ concentrations as functions of space, after 30sec of 5C discharge. Symbols $\{-,+\}$ denotes the negative electrode (anode) and positive electrode (cathode), respectively. In the solid phase concentrations, the top and bottom denote particle center $r=0$ and surface $r=R_{s}^{ \pm}$, respectively.

Along with these equations are corresponding boundary and initial conditions. For brevity, we only summarize the differential equations here. Further details, including notation definitions, can be found in [2], [4]. Parameters for a $\mathrm{LiCoO}_{2}-\mathrm{C}$ cell are publicly available from the DUALFOIL model, developed by Newman and his collaborators [5]. Note the mathematical structure, which contains PDEs (3)-(4), ODEs in time (9), ODEs in space (5)-(7), and nonlinear algebraic constraints (8). This presents a formidable task with respect to control-oriented modeling, state estimation, and control design (see Section III and IV).

\section{Electrochemical Model Simulations}

Illustrative simulations of the electrochemical model are provided in Fig. 5. Specifically, this figure depicts the solid phase $c_{s}^{ \pm}(x, r, t)$ and electrolyte $c_{e}(x, t)$ concentrations as functions of space, after $30 \mathrm{sec}$ of $5 \mathrm{C}^{1}$ discharge. The anode solid phase concentrations $c_{s}^{-}(x, r, t)$ exhibit a decreasing gradient as $r$ increases toward the particle surface, since lithium is exiting the anode during discharge. This creates a spatial gradient in the electrolyte phase $c_{e}(x, t)$ along the $x$-dimension. On the cathode, solid phase concentration increases $c_{s}^{-}(x, r, t)$ w.r.t. $r$, especially near the particle surface, since lithium is entering the cathode.

Note our ultimate goal: estimate these spatio-temporal dynamics and regulate their evolution, given only measurements of voltage, current, and temperature.

\section{Model Reduction}

A rapidly growing body of literature is establishing a spectrum of EChem models that achieve varying balances of mathematical simplicity and accuracy. Concepts include spectral methods [6], [7], residue grouping [8], quasilinearization \& Padé approximation [9], principle orthogonal

${ }^{1} \mathrm{C}$-rate is a normalized measure of electric current that enables comparisons between different sized batteries. Mathematically, the C-rate is defined as the ratio of current, $I$, in Amperes [A] to a cell's nominal capacity, $Q$, in Ampere-hours [Ah]. For example, if a battery has a nominal capacity of $2.5 \mathrm{Ah}$, then $\mathrm{C}$-rates of $2 \mathrm{C}, 1 \mathrm{C}$, and $\mathrm{C} / 2$ correspond to $5 \mathrm{~A}, 2.5 \mathrm{~A}$, and 1.25 $\mathrm{A}$, respectively. Note that $\mathrm{C}$-rate has dimensions of $[\mathrm{A}] /[\mathrm{Ah}]=[1 / \mathrm{h}]$. 
decomposition [10], and single particle model variants [11], [12], [13]. Due to the richness of existing literature, we do not focus on model reduction in this paper. Instead, we focus on the state/parameter estimation and control challenges.

\section{STATE \& PARAMETER ESTIMATION}

\section{A. Survey of SOC/SOH Estimation Literature}

Over the past decade, the $\mathrm{SOC} / \mathrm{SOH}$ estimation literature has grown considerably rich with various algorithms, models, and applications. Most existing literature to date uses equivalent circuit models, but electrochemical model-based estimation algorithms have also emerged. These studies develop estimators for reduced-order models. The model reduction and observer design process are intimately intertwined, as simpler models ease estimation design at the expense of fidelity. Ideally, one seeks to derive a provably stable estimator for the highest fidelity electrochemical battery model possible. The first wave of studies utilized the "single particle model" (SPM) for estimator design [11], [12], [14], [15]. The SPM idealizes each electrode as a single spherical porous particle by neglecting the electrolyte dynamics. This model is suitable for low C-rates, but degrades at C-rates above $\mathrm{C} / 2$. Recently, researchers have extended the single particle model to include electrolyte dynamics [13], [16], although there have been limited estimation studies [17], [18]. Nonetheless, state estimation designs have emerged for other electrochemical models that incorporate electrolyte dynamics. Examples include spectral methods with output injection [19], residue grouping with Kalman filtering [20], and composite electrodes with nonlinear filters [21]. Unfortunately, it becomes more difficult to prove estimation error stability as model complexity increases. The core difficulty is lack of complete observability from voltage measurements.

\section{B. Single Particle Model (SPM)}

To illustrate the control-theoretic challenges of $\mathrm{SOC} / \mathrm{SOH}$ estimation, we consider the SPM - the simplest form of electrochemical model. The SPM idealizes each electrode as a single aggregate spherical particle. One can derive this model by assuming the electrolyte Li concentration $c_{e}(x, t)$ from (4) is constant in space and time. Mathematically, the model consists of two diffusion PDEs,

$$
\begin{array}{r}
\frac{\partial c_{s}^{ \pm}}{\partial t}(r, t)=D_{s}^{ \pm}\left[\frac{2}{r} \frac{\partial c_{s}^{ \pm}}{\partial r}(r, t)+\frac{\partial^{2} c_{s}^{ \pm}}{\partial r^{2}}(r, t)\right], \\
\frac{\partial c_{s}^{ \pm}}{\partial t}(0, t)=0, \quad \frac{\partial c_{s}^{ \pm}}{\partial t}\left(R_{s}^{ \pm}, t\right)=\frac{ \pm I(t)}{D_{s}^{ \pm} F a^{ \pm} A L^{ \pm}} .
\end{array}
$$

The boundary conditions at $r=R_{s}^{+}$and $r=R_{s}^{-}$signify that flux is proportional to input current $I(t)$. Output voltage is given by a nonlinear function of the state values at the boundary $c_{s s}^{+}(t), c_{s s}^{-}(t)$ and the input current $I(t)$ as follows

$$
\begin{aligned}
V(t)= & \frac{R T}{\alpha F} \sinh ^{-1}\left(\frac{-I(t)}{2 a^{+} A L^{+} i_{0}^{+}\left(c_{s s}^{+}(t) ; n_{L i}\right)}\right) \\
& -\frac{R T}{\alpha F} \sinh ^{-1}\left(\frac{I(t)}{2 a^{-} A L^{-} i_{0}^{-}\left(c_{s s}^{-}(t) ; n_{L i}\right)}\right) \\
& +U^{+}\left(c_{s s}^{+}(t)\right)-U^{-}\left(c_{s s}^{-}(t)\right)+R_{f} I(t),
\end{aligned}
$$

$$
V(t)=h\left(c_{s s}^{-}, c_{s s}^{+}, I ; \theta\right),
$$

where the $i_{0}^{j}(\cdot)$ is the exchange current density and $c_{s s}^{j}(t)=$ $c_{s}^{j}\left(R_{s}^{j}, t\right)$ is the surface concentration for electrode $j \in$ $\{+,-\}$. The functions $U^{j}(\cdot)$ are the equilibrium potentials of each electrode material, given the surface concentration. Mathematically, the functions $U^{j}(\cdot)$ are strictly monotonically decreasing functions of their input. This fact implies the inverse of its derivative is always finite, a property that is critically important for assessing observability. Symbol $n_{L i}$ represents the total moles of cyclable lithium. Finally, parameter vector $\theta=\left[n_{L i},\left(a^{+} A L^{+} k^{+}\right)^{-1},\left(a^{-} A L^{-} k^{-}\right)^{-1}, R_{f}\right]^{T}$ represents uncertain parameters.

Remark 1 (SOH Metrics): Coincidently, the parameters $n_{L i}$ and $R_{f}$ represent capacity and impedance rise, respectively. Identification of $n_{L i}$ and $R_{f}$ provides a direct system-level estimate of $\mathrm{SOH}$.

Combined SOC/SOH Problem: Given measurements of current $I(t)$, voltage $V(t)$, and the SPM equations, simultaneously estimate the lithium concentration states $c_{s}^{-}(r, t), c_{s}^{+}(r, t)$ and parameters $\theta$.

\section{Model Properties}

Before embarking on an estimation design, we first study relevant mathematical properties of the SPM. The $c_{s}^{+}, c_{s}^{-}$ subsystems are mutually independent of each other. Moreover, they are governed by linear PDEs. Also note that the PDE subsystems produce boundary values $c_{s s}^{+}(t), c_{s s}^{-}(t)$ that feed into the nonlinear output function (17). The SPM is also characterized by the following dynamical properties, which present notable challenges for state estimation. We present the following propositions, whose proofs are straightforward, non-insightful, and omitted for brevity.

Proposition 1 (Marginal Stability): Each individual subsystem in (15)-(16) governing states $c_{s}^{+}(r, t), c_{s}^{-}(r, t)$ is marginally stable. In particular, each subsystem contains one eigenvalue at the origin, and the remaining eigenvalues lie on the negative real axis of the complex plane.

Proposition 2 (Conservation of Lithium): The moles of lithium in the solid phase are conserved [19]. Mathematically, $\frac{d}{d t}\left(n_{L i}(t)\right)=0$ where

$$
n_{L i}(t)=\sum_{j \in\{+,-\}} \frac{\epsilon_{s}^{j} L^{j} A}{\frac{4}{3} \pi\left(R_{s}^{j}\right)^{3}} \int_{0}^{R_{s}^{j}} 4 \pi r^{2} c_{s}^{j}(r, t) d r
$$

Invertability Analysis: Next, we study invertability of the output function (17) w.r.t. boundary state variables $c_{s s}^{ \pm}(t)$. Figure 6(a) provides the open circuit potential (OCP) functions $U^{-}(\cdot), U^{+}(\cdot)$ as functions of the normalized anode concentration $\theta^{ \pm}=c_{s s}^{ \pm} / c_{s, \text { max }}^{ \pm}$. The near zero gradient implies the voltage measurement is weakly sensitive to perturbations in the surface concentrations $c_{s s}^{ \pm}$. This is further confirmed by Fig. 6(b), which depicts the output function's partial derivatives w.r.t. $c_{s s}^{-}$and $c_{s s}^{+}$at equilibrium conditions, for currents ranging from $-5 \mathrm{C}$ to $+5 \mathrm{C}$. It is important to note that $h$ is strictly monotonically decreasing w.r.t. $c_{s s}^{+}$over a larger range than $h$ is strictly monotonically increasing w.r.t. 


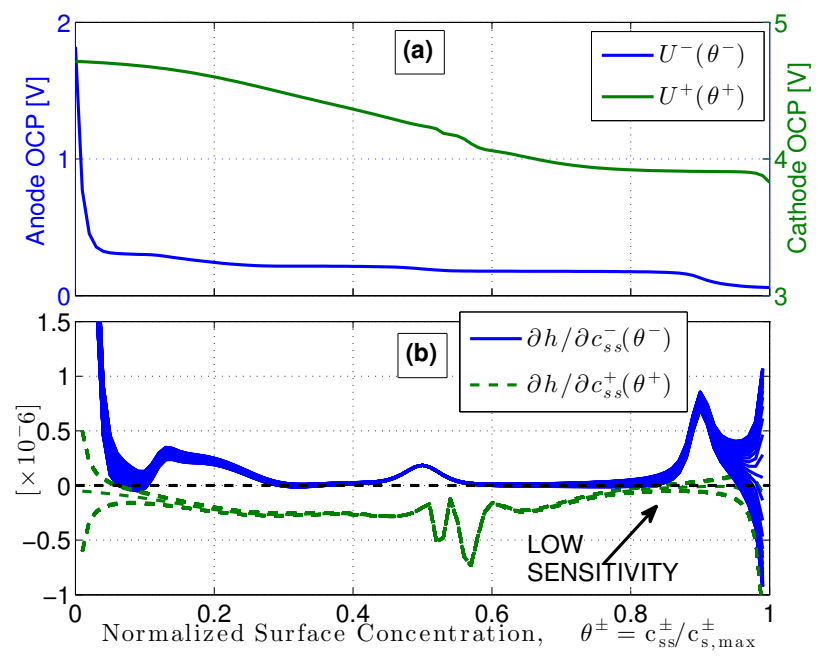

Fig. 6. (a) Open circuit potential functions $U^{-}\left(\theta^{-}\right), U^{+}\left(\theta^{+}\right)$; and (b) Gradients of $h\left(c_{s s}^{+}, c_{s s}^{-}, I\right)$ in (18), as functions of normalized surface concentration $\theta^{ \pm}=c_{s s}^{ \pm} / c_{s, \max }^{ \pm}$, for currents ranging from $-5 \mathrm{C}$ to $+5 \mathrm{C}$.

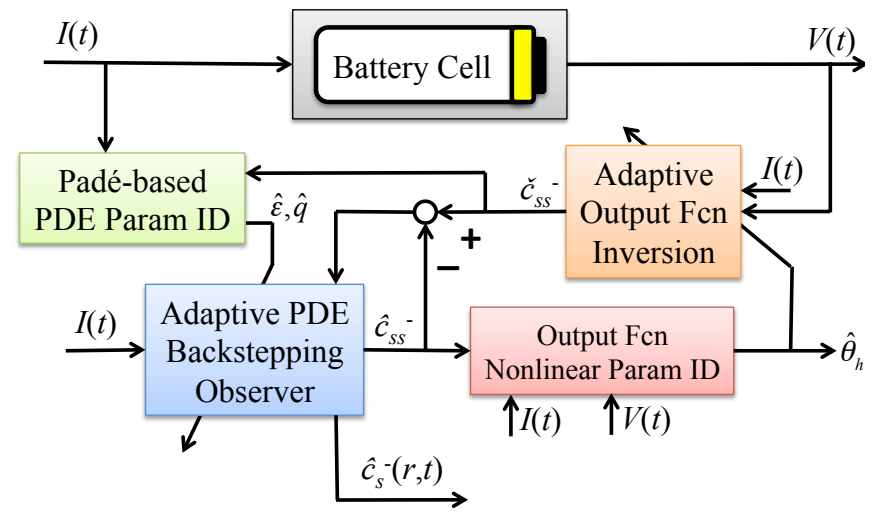

Fig. 7. Block diagram of Adaptive PDE Observer for SOC/SOH Estimation. See [14] for details.

$c_{s s}^{-}$. This property is critical, since it demonstrates that voltage is generally more sensitive to perturbations in cathode surface concentration than anode surface concentration. Note $\partial h / \partial c_{s s}^{+} \approx 0$ for $0.8 \leq \theta^{+} \leq 0.9$. This region is a "blind spot" with respect to output inversion.

Nonlinear Parameter Sensitivity Analysis: Since the parameter vector $\theta$ enters nonlinearly in the output function (18), we must first perform a sensitivity analysis to assess linear dependence. Define the sensitivity vector $S=\partial h / \partial \theta$. Applying the ranking procedure outlined in [22] to $S^{T} S$ reveals strong linear dependence exists between $\theta_{2}, \theta_{3}, \theta_{4}$, where $\theta_{4}$ exhibits the greatest sensitivity magnitude. As a result, we pursue a parameter identification scheme for the parameter subset $\left(\theta_{1}, \theta_{4}\right)$. Coincidentally, these two parameters are exactly $n_{L i}, R_{f}$, corresponding to capacity fade and impedance rise. Refer to [14] for complete details.

\section{Adaptive PDE Observer Approach}

The previous subsection described several fundamental estimation challenges for the SPM, including marginal stability, poor output sensitivity to states, and linear dependence between parameters. An adaptive PDE observer was designed for this model in [14], which achieves stable estimates by
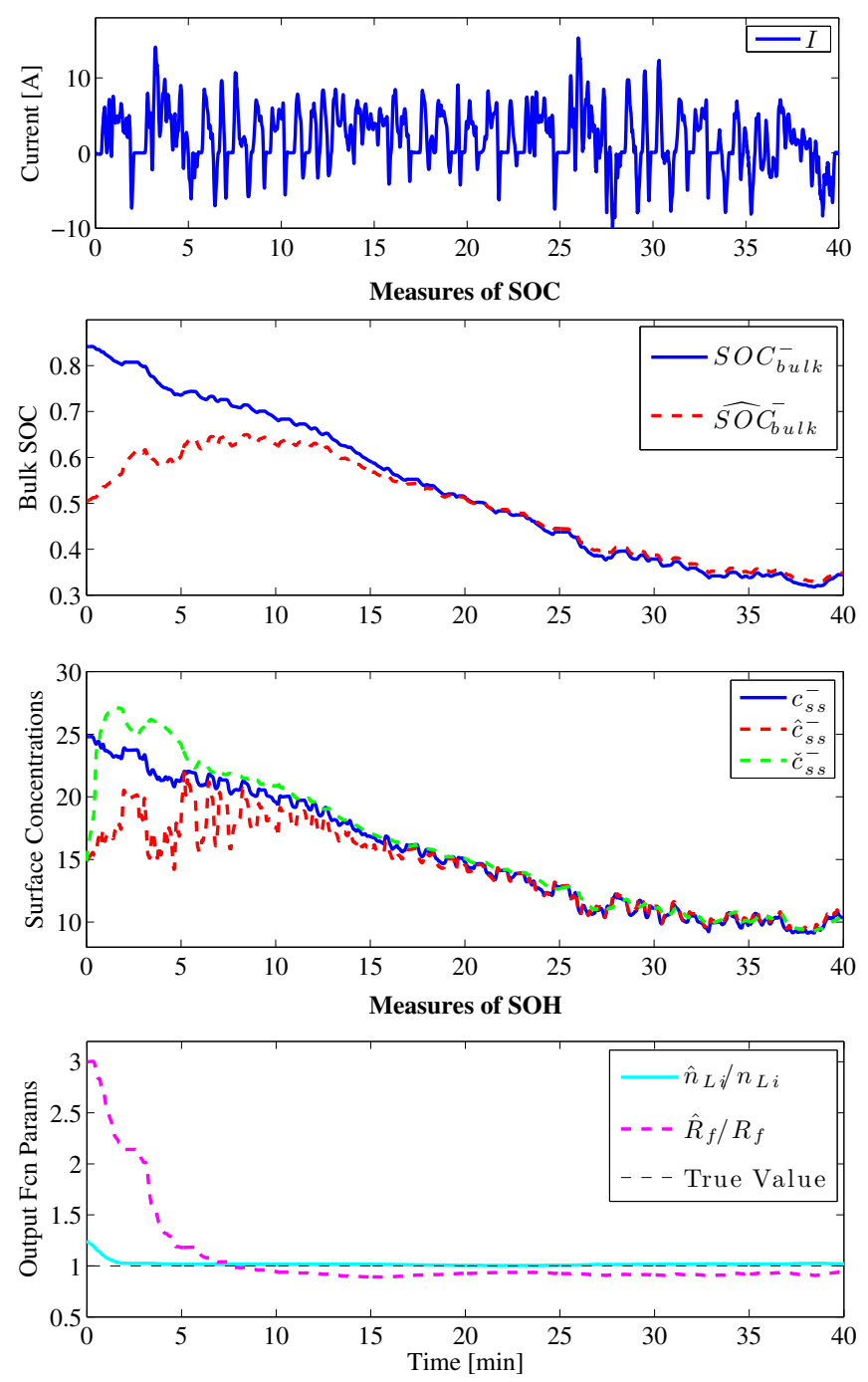

Fig. 8. Evolution of state and parameter estimates from Adaptive PDE observer [14] for UDDSx 2 charge/discharge cycle. Zero mean Gaussian noise with a $10 \mathrm{mV}$ variance was added to the voltage measurement. The EChem model (3)-(14) provides the "measured" plant data.

exploiting output function invertibility and lithium conservation. Figure 7 summarizes this scheme. The algorithm is composed of an Adaptive PDE backstepping observer [23], Padé-based parameter identifier for PDE parameters [9], nonlinear recursive least squares identifier for output function parameters, and an adaptive output function inversion scheme [24]. A simulation example is provided in Fig. 8, which demonstrates SOC and $\mathrm{SOH}$ convergence given incorrect initial estimates. We remark that the fundamental challenges for estimation are significant, even for the simplest of EChem models - the SPM.

\section{CONSTRAINED OPTIMAL CONTROL}

\section{A. Survey of Optimal Battery Charge/Discharge Literature}

A rich body of literature exists on battery charging/discharging performance. Interestingly, the bulk of research involves experimental studies and non-model based heuristic charging protocols (see [25] for a survey). Relatively speaking, research on electrochemical model-based 
TABLE I

ELECTROCHEMICAL VARIABLES $y$ TO REGULATE WITHIN BOX CONSTRAINTS, I.E. $y_{\min } \leq y \leq y_{\max }$.

\begin{tabular}{|c|c|c|}
\hline Variable, $y$ & Definition & Constraint \\
\hline$I(t)$ & Current & Power electronics limit \\
\hline$c_{s}^{ \pm}(x, r, t)$ & Li concentration in solid & $\begin{array}{l}\text { Material } \\
\text { saturation/depletion }\end{array}$ \\
\hline$\frac{\partial c_{s}^{ \pm}}{\partial r}(x, r, t)$ & $\begin{array}{l}\text { Li concentration gradient } \\
\text { in solid }\end{array}$ & $\begin{array}{l}\text { Diffusion-induced } \\
\text { stress/strain }\end{array}$ \\
\hline$c_{e}(x, t)$ & $\begin{array}{l}\mathrm{Li} \text { concentration in } \\
\text { electrolyte }\end{array}$ & $\begin{array}{l}\text { Material } \\
\text { saturation/depletion }\end{array}$ \\
\hline$T(t)$ & Temperature & $\begin{array}{l}\text { High/low temperatures } \\
\text { accelerate aging }\end{array}$ \\
\hline$\eta_{s}(x, t)$ & $\begin{array}{l}\text { Side reaction } \\
\text { overpotential }\end{array}$ & $\begin{array}{l}\text { Li-plating, SEI-layer } \\
\text { growth }\end{array}$ \\
\hline
\end{tabular}

control is nascent. Existing optimal control studies include constrained control [20], [26] and open-loop control [27], [28], [29]. A recent study by the author performs a comprehensive assessment of performance improvements enabled by reference governors and electrochemical models [30].

\section{B. A Reference Governor Approach}

Ensuring safe operating constraints is a basic requirement for batteries. Mathematically, this can be abstracted as a constrained control problem for which reference governors provide one effective solution. The problem statement is:

Constrained Control Problem: Given accurate state/parameter estimates $(\hat{x}, \hat{\theta})$, regulate input current $I(t)$ such that the EChem constraints in Table I are enforced pointwise in time (see Fig. 2).

We seek to maintain operation subject to electrochemical state constraints. This protects the battery against catastrophic failure and maintains longevity. A list of relevant state constraints is provided in Table I. These limits are associated with material saturation/depletion, mechanical stress, extreme temperatures, and harmful side reactions, such as lithium plating and solid/electrolyte interphase film growth.

A reference governor is an add-on algorithm that guarantees constraint satisfaction pointwise-in-time while tracking a desired reference input [31], [32]. In our "modified" reference governor (MRG) implementation, the applied current $I[k]$ and reference current $I^{r}[k]$ are related by

$$
I[k+1]=\beta[k] I^{r}[k], \quad \beta \in[0,1],
$$

where $I[k]=I(t)$ for $t \in[k \Delta t,(k+1) \Delta t), k \in \mathbb{Z}$. The goal is to maximize $\beta$ such that the state stays within an admissible set $\mathcal{O}$ over some future time horizon,

$$
\beta^{*}[k]=\max \{\beta \in[0,1]: x(t) \in \mathcal{O}\} .
$$

Variable $x(t)$ represents the electrochemical model state at time $t$ and $\mathcal{O}$ is the set of initial conditions that maintain the state within the constraints listed in Table I, over a future time horizon $\tau \in\left[t, t+T_{s}\right]$ [30].

We evaluate the power and energy capacity benefits of the MRG versus an industry standard Voltage-Only (VO) controller on electric vehicle-like charge/discharge cycles.
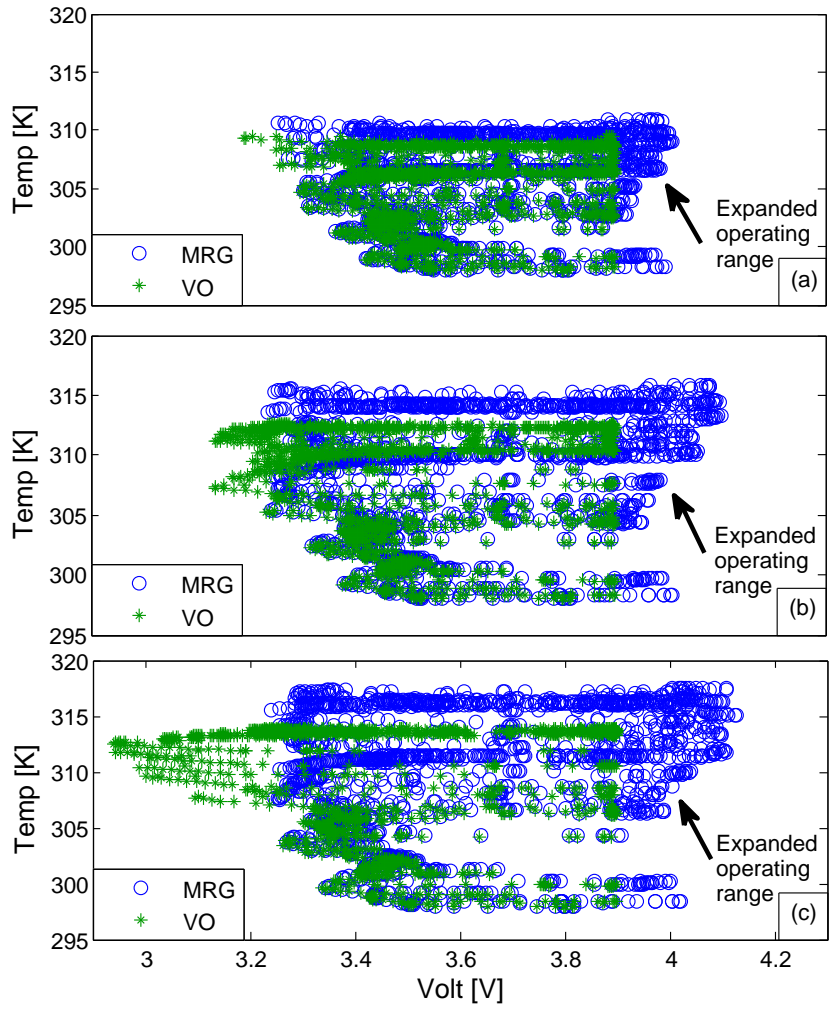

Fig. 9. Temperature vs. Voltage operating points for (a) 1.0I, (b) 1.2I, and (c) $1.4 \mathrm{I}$ over US06x3 cycle.

The VO governor is structurally equivalent to the MRG, except the constraints in Table I are replaced by voltage limits of $2.8 \mathrm{~V}$ and $3.9 \mathrm{~V}$. Various automotive-relevant charge/discharge cycles cases were tested. To explore state constraint management, reference current was scaled by factors of $\times 1.0, \times 1.2, \times 1.4(1.0 \mathrm{I}, 1.2 \mathrm{I}, 1.4 \mathrm{I})$. Due to space constraints, we only provide examples with three concatenated US06 drive cycles (US06x3).

Figure 9 depicts the Temperature vs. Voltage operational points for the MRG vs. VO controllers for the US06x3 1.0I, 1.2I, and 1.4I current profiles. The VO controller upper voltage limit becomes more constrictive as the current multiplier increases. The MRG safely exceeds the VO voltage limits without violating the electrochemical constraints. In automotive applications, this ultimately means the MRG recuperates more energy (i.e. from regenerate braking) than the VO controller. We numerically compared the MRG and VO across five other automotive drive cycles from [33]. In the most aggressive drive cycle (US06x3) the MRG achieves $11.03 \%$ and $150.61 \%$ more discharge and charge power, respectively, over the VO controller (1.4I case). The MRG also achieves a $22.99 \%$ net energy increase. Across all six simulated drive cycles, the MRG achieves average increases in discharge power, charge power, and net energy of $4.92 \%, 57.15 \%$, and $10.04 \%$, respectively (1.4I case). See [30] for complete details. Consequently, we conclude that electrochemical model based control enables substantial performance improvements, provided the electrochemical states and parameters can be accurately estimated. 


\section{CONCLUSIONS}

This paper provides a tutorial on estimation and control challenges for electrochemical battery models. We first provide a brief history of the electrochemical battery cell, and then discuss the fundamental operating principles. Next, we present concrete statements for the state-of-charge (SOC), state-of-health ( $\mathrm{SOH})$, and charge/discharge control problems. Mathematically, these are respectively cast as state estimation, parameter estimation, and constrained control problems. We discuss combined SOC/SOH estimation and highlight some solutions and several challenges. Finally, we discuss constrained control via modified reference governors.

Electrochemical models provide an exceptionally rich application for challenging systems and controls problems. The combined state/parameter estimation problem is particularly difficult. Ultimately, our objective is to derive provably stable estimators for increasingly complex electrochemical models. For example, recent work extends the SPM to include electrolyte dynamics [13], [18], temperature [16], and multiple material cathodes [21]. Fundamental challenges include model reduction, observability, identifiability, nonlinearity, spatio-temporal dynamics, and numerical implementations.

\section{REFERENCES}

[1] M. Armand and J.-M. Tarascon, "Building better batteries," Nature, vol. 451 , no. 7179 , pp. $652-657,2008$.

[2] N. A. Chaturvedi, R. Klein, J. Christensen, J. Ahmed, and A. Kojic, "Algorithms for advanced battery-management systems," IEEE Control Systems Magazine, vol. 30, no. 3, pp. 49 - 68, 2010.

[3] G. Pancaldi, Volta: Science and culture in the age of enlightenment. Princeton University Press, 2005.

[4] K. Thomas, J. Newman, and R. Darling, Advances in Lithium-Ion Batteries. New York, NY USA: Kluwer Academic/Plenum Publishers, 2002, ch. 12: Mathematical modeling of lithium batteries, pp. 345392.

[5] J. Newman. (2008) Fortran programs for the simulation of electrochemical systems. [Online]. Available: http://www.cchem. berkeley.edu/jsngrp/fortran.html

[6] V. R. Subramanian, V. Boovaragavan, V. Ramadesigan, and M. Arabandi, "Mathematical model reformulation for lithium-ion battery simulations: Galvanostatic boundary conditions," Journal of the Electrochemical Society, vol. 156, no. 4, pp. A260 - A271, 2009.

[7] C. Mayhew, W. He, C. Kroener, R. Klein, N. Chaturvedi, and A. Kojic, "Investigation of projection-based model-reduction techniques for solid-phase diffusion in li-ion batteries," in 2014 American Control Conference (ACC), June 2014, pp. 123-128.

[8] K. A. Smith, C. D. Rahn, and C.-Y. Wang, "Model order reduction of $1 \mathrm{~d}$ diffusion systems via residue grouping," ASME Journal of Dynamic Systems, Measurement, and Control, vol. 130, no. 1, p. 011012, 2008.

[9] J. C. Forman, S. Bashash, J. L. Stein, and H. K. Fathy, "Reduction of an electrochemistry-based li-ion battery model via quasi-linearization and pade approximation," Journal of the Electrochemical Society, vol. 158, no. 2, pp. A93 - A101, 2011.

[10] L. Cai and R. E. White, "Reduction of model order based on proper orthogonal decomposition for lithium-ion battery simulations," Journal of The Electrochemical Society, vol. 156, no. 3, pp. A154-A161, 2009.

[11] S. Santhanagopalan and R. E. White, "Online estimation of the state of charge of a lithium ion cell," Journal of Power Sources, vol. 161, no. 2, pp. $1346-1355,2006$.

[12] D. D. Domenico, A. Stefanopoulou, and G. Fiengo, "Lithium-Ion Battery State of Charge and Critical Surface Charge Estimation Using an Electrochemical Model-Based Extended Kalman Filter," Journal of Dynamic Systems, Measurement, and Control, vol. 132, no. 6, p. 061302, 2010.

[13] S. K. Rahimian, S. Rayman, and R. E. White, "Extension of physicsbased single particle model for higher charge-discharge rates," Journal of Power Sources, vol. 224, no. 0, pp. 180 - 194, 2013.
[14] S. J. Moura, N. Chaturvedi, and M. Krstic, "Adaptive PDE Observer for Battery SOC/SOH Estimation via an Electrochemical Model," ASME Journal of Dynamic Systems, Measurement, and Control, vol. 136, no. 1, pp. 011015-011026, Oct 2014.

[15] S. Dey and B. Ayalew, "Nonlinear observer designs for state-ofcharge estimation of lithium-ion batteries," in 2014 American Control Conference (ACC), June 2014, pp. 248-253.

[16] T. Tanim, C. Rahn, and C.-Y. Wang, "A reduced order electrolyte enhanced single particle lithium ion cell model for hybrid vehicle applications," in 2014 American Control Conference (ACC), June 2014, pp. 141-146.

[17] T. R. Tanim, C. D. Rahn, and C.-Y. Wang, "State of charge estimation of a lithium ion cell based on a temperature dependent and electrolyte enhanced single particle model," Energy, vol. 80, no. 0, pp. 731 - 739, 2015.

[18] X. Han, M. Ouyang, L. Lu, and J. Li, "Simplification of physics-based electrochemical model for lithium ion battery on electric vehicle. Part II: Pseudo-two-dimensional model simplification and state of charge estimation," Journal of Power Sources, vol. 278, no. 0, pp. 814 - 825, 2015.

[19] R. Klein, N. A. Chaturvedi, J. Christensen, J. Ahmed, R. Findeisen, and A. Kojic, "Electrochemical Model Based Observer Design for a Lithium-Ion Battery," IEEE Transactions on Control Systems Technology, vol. 21, no. 2, pp. 289-301, March 2013.

[20] K. A. Smith, C. D. Rahn, and C.-Y. Wang, "Model-based electrochemical estimation and constraint management for pulse operation of lithium ion batteries," IEEE Transactions on Control Systems Technology, vol. 18, no. 3, pp. $654-663,2010$.

[21] A. Bartlett, J. Marcicki, S. Onori, G. Rizzoni, X. Yang, and T. Miller, "Electrochemical model-based state of charge and capacity estimation for a composite electrode lithium-ion battery," IEEE Transactions on Control Systems Technology, vol. PP, no. 99, pp. 1-1, 2015.

[22] B. F. Lund and B. A. Foss, "Parameter ranking by orthogonalization Applied to nonlinear mechanistic models," Automatica, vol. 44, no. 1, pp. $278-281,2008$.

[23] A. Smyshlyaev and M. Krstic, "Backstepping observers for a class of parabolic PDEs," Systems \& Control Letters, vol. 54, no. 7, pp. 613-625, 2005.

[24] P. Ioannou and J. Sun, Robust Adaptive Control. Prentice-Hall, 1996.

[25] S. S. Zhang, "The effect of the charging protocol on the cycle life of a li-ion battery," Journal of power sources, vol. 161, no. 2, pp. 1385-1391, 2006.

[26] R. Klein, N. A. Chaturvedi, J. Christensen, J. Ahmed, R. Findeisen, and A. Kojic, "Optimal charging strategies in lithium-ion battery," in American Control Conference, San Francisco, CA, United states, 2011, pp. $382-387$.

[27] R. Methekar, V. Ramadesigan, R. D. Braatz, and V. R. Subramanian, "Optimum charging profile for lithium-ion batteries to maximize energy storage and utilization," ECS Transactions, vol. 25, no. 35, pp. 139-146, 2010.

[28] S. Bashash, S. J. Moura, J. C. Forman, and H. K. Fathy, "Plug-in hybrid electric vehicle charge pattern optimization for energy cost and battery longevity," Journal of Power Sources, vol. 196, no. 1, pp. 541 $-549,2011$.

[29] X. Hu, H. Perez, and S. J. Moura, "Battery charge control with an electro-thermal-aging coupling," in 2015 ASME Dynamic Systems and Control Conference, Columbus, $\mathrm{OH}, 2015$.

[30] H. Perez, N. Shahmohammadhamedani, and S. Moura, "Enhanced performance of li-ion batteries via modified reference governors and electrochemical models," IEEE/ASME Transactions on Mechatronics, vol. 20, no. 4, pp. 1511-1520, Aug 2015.

[31] E. Gilbert, I. Kolmanovsky, and K. Tan, "Discrete-time reference governors and the nonlinear control of systems with state and control constraints," International Journal of Robust and Nonlinear Control, vol. 5, no. 5, pp. 487-504, 1995.

[32] I. Kolmanovsky, E. Garone, and S. Di Cairano, "Reference and command governors: A tutorial on their theory and automotive applications," in American Control Conference (ACC), 2014, June 2014, pp. 226-241.

[33] S. Moura, J. Stein, and H. Fathy, "Battery-Health Conscious Power Management in Plug-In Hybrid Electric Vehicles via Electrochemical Modeling and Stochastic Control," IEEE Transactions on Control Systems Technology, vol. 21, no. 3, pp. 679-694, 2013. 